
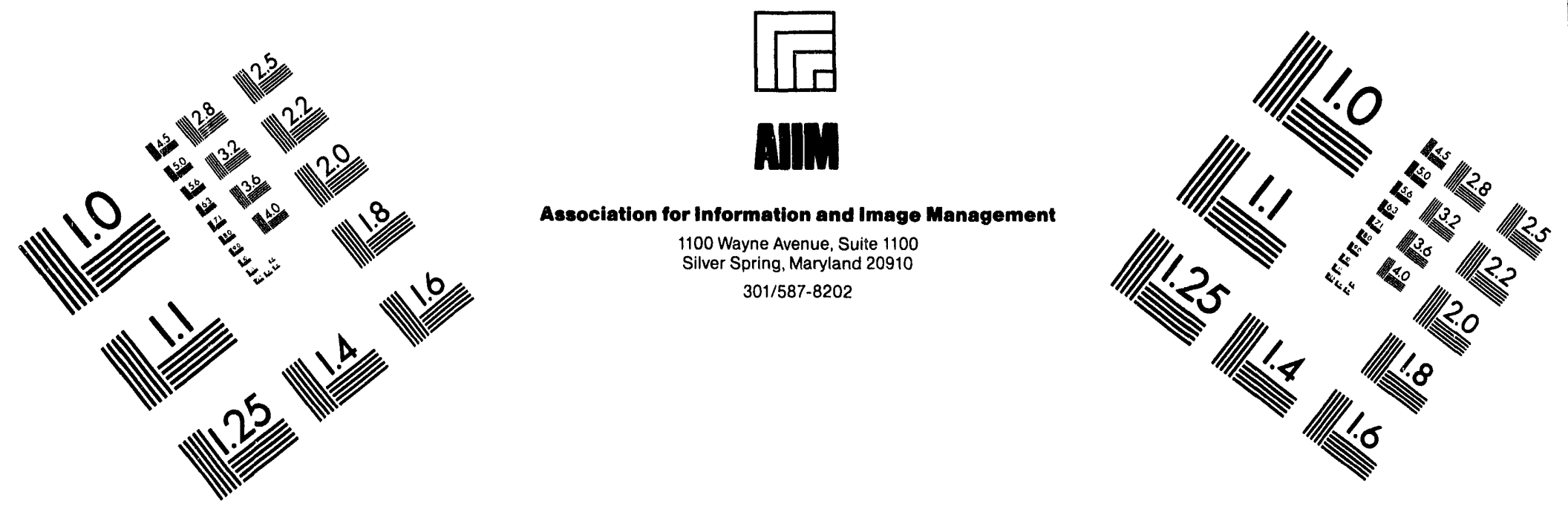

Centimeter

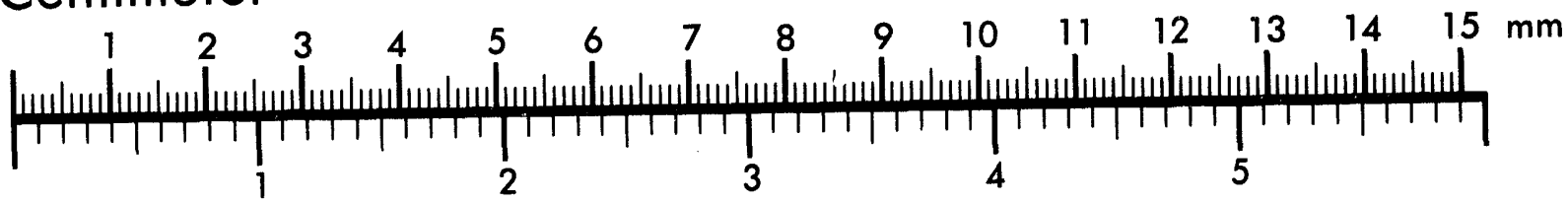
Inches
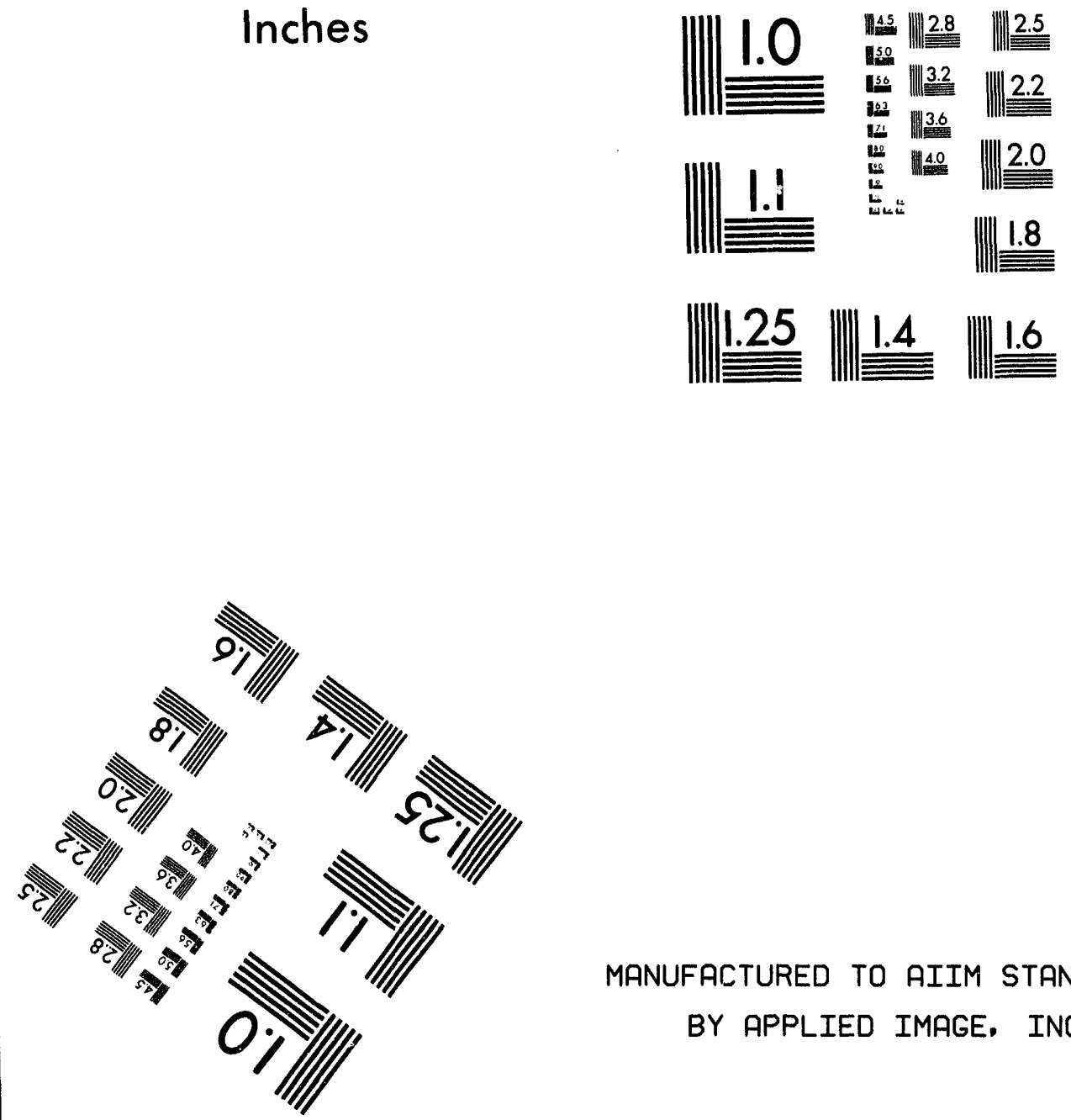

MANUFACTURED TO AIIM STANDARDS

BY APPLIED IMAGE, INC.

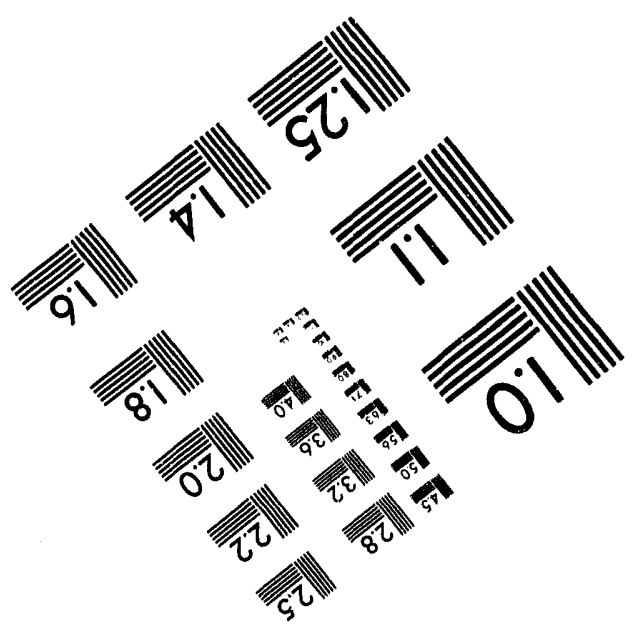



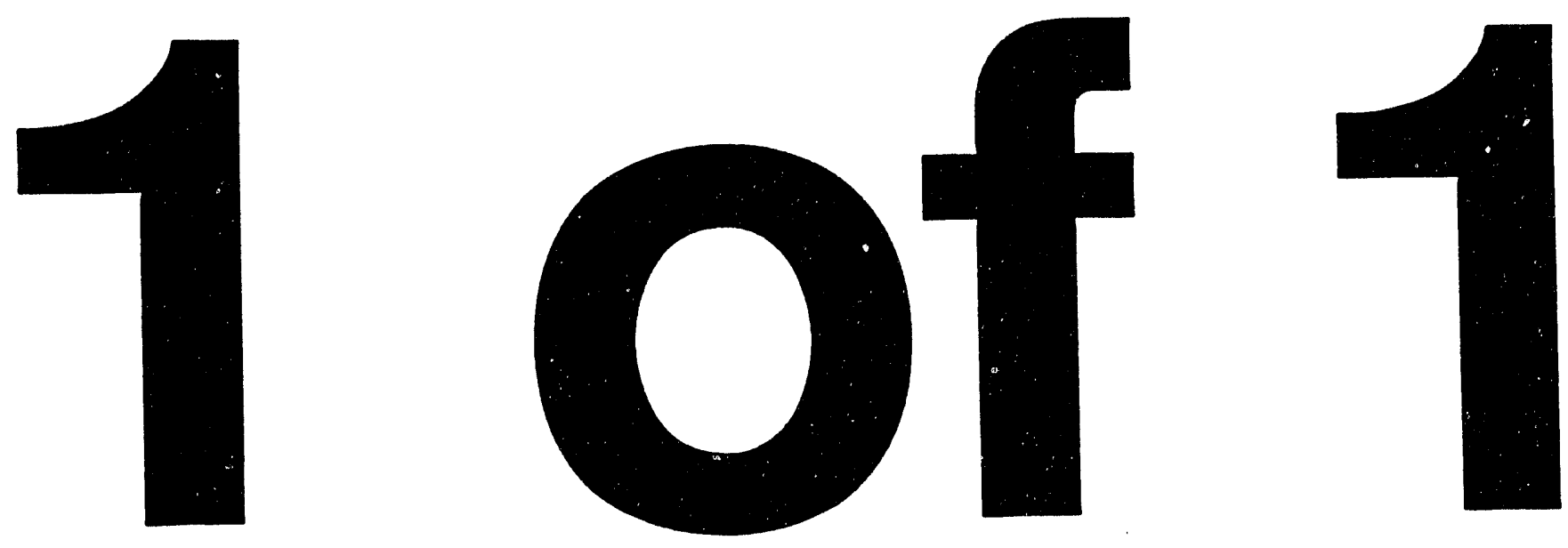


\title{
TITLE: REGENERATOR OPTIMIZATION FOR STIRLING CYCLE
} RERIGERATION II

\author{
AUTHOR(S): S. Colgate and A. Petschek, T-6
}

SUBMITTED TO: The 8th International Conference on Cryocooler held in Vail, CO 6/28-6/30, 1994.

\section{DISCLAIMER}

This report was prepared as an account of work sponsored by an agency of the United States Government. Neither the United States Government nor any agency thereof, nor any of their employees, makes any warranty, express or implied, or assumes any legal liability or responsibility for the accuracy, completeness, or usefulness of any information, apparatus, product, or process disclosed, or represents that its use would not infringe privately owned rights. Reference herein to any specific commercial product, process, or service by trade name, trademark, manufacturer, or otherwise does not necessarily constitute or imply its endorsement, recommendation, or favoring by the United States Government or any agency thereof. The views and opinions of authors expressed herein do not necessarily state or reflect those of the United States Government or any agency thereof.
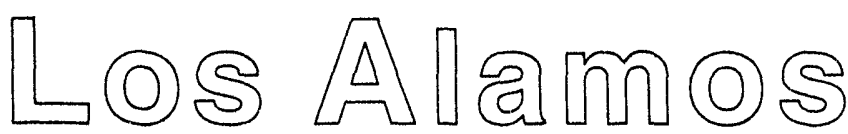

Los Alamos National Laboratory Los Alamos, New Mexico 87545 


\title{
REGENERATOR OPTIMIZATION FOR STIRLING CYCLE REFRIGERATION II
}

\author{
Stirling A. Colgate and Albert G. Petschek \\ Los Alamos National Laboratory, Los Alamos, NM 87545
}

\begin{abstract}
A cryogenic regenerator for a Stirling cycle is discussed using fractional loss or entropy gain as the criterion of performance. The gas losses are treated separately from heat storage medium losses. We argue that the optimum design corresponds to uniform channel flow with minimum turbulence where the gas velocity and channel width are optimized as a function of gas temperature. The maximization of heat transfer from the gas to the wall and the minimization of entropy production by friction leads to a gas flow velocity equal to sound speed times loss fraction, $1 / \sigma$. This velocity and an axial thermal conductivity in the gas leads to a minimum channel width and characteristic length, $\mathrm{L}=\mathrm{T}(\mathrm{dz} / \mathrm{dT})$. A particular scaling of width, $\mathrm{W}^{2}=$ $\mathrm{W}_{0} \mathrm{~T}^{1 / 2}$, and length, $\mathrm{L}=\mathrm{L}_{0} \mathrm{~T}^{-1 / 2}$ leads to a design where longitudinal conduction decreases as $\mathrm{T}^{3 / 2}$ and the remaining two losses, transverse conduction and friction are equal and constant. The loss fraction, $1 / \sigma$, must be made quite small, $\sim(1 / 60)$ in order that the cumulative losses for a large temperature ratio like $300 \mathrm{~K}$ to $4 \mathrm{~K}$, be small enough, like $20 \%$ to $40 \%$. This is because half the entropy generated as a loss must be transported first to the cold end before returning to the hot end before being rejected. The dead volume ratio then determines the minimum frequency and with it and the pressure the necessary wall properties. The thermal properties of the channel wall must then accommodate this cyclic heat flow without substantially increasing the loss fraction. This generation of entropy in the walls is derived in terms of the wall heat capacity and thermal conductivity
\end{abstract}

\section{Introduction}

Regenerators in Stirling refrigeration store and return the heat of the working fluid, a gas, during each of two phases of the cycle. The design objective, for high efficiency, is to transfer a given heat mass from one temperature to another with the minimum change in entropy. If the temperature difference of the refrigerator is small, relative to absolute temperature, as in domestic heat pumps, the regenerator is simpler to design because the thermal properties of the materials and of the gas remain nearly constant throughout tise regenerator. In cryogenic refrigerators where the temperature ratio is large, and where one hopes to achieve the low temperature without intermediate expansion and compression volumes, the regenerator is more complex and the efficiency more sensitive to its properties.

In the previous paper (Colgate and Petschek, 1992, hereafter CP I) we addressed primarily the efficiency of the regenerator as determined by the gas properties. This led to 
several relationships between dimensions, velocity, gas properties and losses. The wall properties were not considered and hence were assumed ideal. In this paper we will address first the overall efficiency as determined by the integral effect of many infinitesimal stages or sections

of regenerator in series. Knowing the efficiency desired as a function of temperature and the dimensions and velocities that result in the desired gas efficiency, we will then consider the problem of the wall materials that satisfy the gas boundary conditions. There are, however, several important additional components to a cryocooler that will affect its efficiency. We assume that our regenerator is bounded at each end by isothermal compression and expansion volumes. These volumes will have their own losses although we expect that the use of special metal bellows (Colgate 1986) can keep these losses small. Similarly, radiation and conduction to the regenerator as a function of length and hence, temperature should be intercepted at as high a temperature as possible so that the entropy gain will be a minimum.

Sullivan, Radebaugh, Daney, and Zimmerman (1982) considered a similar design problem, but did not include the entropy gain from the frictional heat of the gas flow. With the inclusion of this loss, the geometry restrictions are altered. In addition we will consider the dead volume of the regenerator as one constraint because it affects the losses as well as the useful refrigeration. Minimizing the dead volume along with the entropy gain will dictate the channel geometry of the regenerator.

In Section II we will review the assumptions of CP I with initial emphasis on the question of why we choose a smooth wall channel of nearly constant width instead of the frequently used geometries of wire mesh or spheres of heat mass material. In addition, we will review the effect on overall efficiency of staging where before we divided conceptually the regenerator into discreet stages and now consider a continuum of properties. We derive the limiting loss for this continuum and thus establish a goal for the desired efficiency at every point of the design.. We will then review from CP I each of the gas losses using as the non-dimensional loss, the fractional addition of entropy per unit of entropy transferred. This allows one to relate frequency, channel length, temperature, dead volume ratio, and non dimensional loss.

In Section III using this desired efficiency we consider a design of the gas flow geometry that optimizes the dead volume and therefore the geometry in the cold end where material properties are most limiting.

In section IV we consider the desired properties of wall materials and by considering how the heat flow within these materials adds to the losses that occur purely within them. Section V is the conclusion.

\section{Regenerator Gas Efficiency}

\section{Channel Geometry}

Classically, regenerators in Stirling cycle heat pumps are heterogeneous, typically made of wire mesh, spheres, or random wires (see Walker 1980). Radebaugh and Louie (1984) and independently Colgate (1986) have pointed out that the smooth wall channel is a far better geometry for efficient heat transfer. This is based upon the extensive measurements of transient heat flow by Coopage and London (1956). A quantitative comparison is complex and given in the above references. The additional dead volume penalty for a random matrix regenerator giving the same heat transfer and friction loss compared to a uniform width channel is large, $>10$ but this has not yet been proven experimentally (Radebaugh, 1992, pvt. communication). We proceed with a design based upon uniform channel flow with the expectation of performing such a comparison. A qualitative argument is that if one channel width is optimum, a different one is not. A heterogeneous material defines a channel that varies in width, and so cannot be optimum. It results in an increased dead volume, which causes an unnecessary cycle loss.

In addition one questions whether turbulence induced by the irregularities will be an advantage. If eddy motion decayed only by friction with the walls, then the effect of turbulence might possibly be beneficial. However, the Prandtl number (ratio of thermal to viscous diffusivity) is reduced from 1.66 for a monatomic gas, i.e. helium, to 1.0 for turbulence, i.e. an eddy transports heat and momentum macroscopically and thus equally. Thus the particular advantage of larger thermal diffusivity is lost. We therefore choose channel flow as the optimum regenerator configuration.

Regenerator Section Independence 
For practical reasons of material properties and fabrication, in CP I we imagined sections of one given wall material and constant channel width for a finite temperature difference of an individual section. In terms of the coefficient of performance (COP) of one stage and its Carnot value (CCOP) we define a normalized COP (NCOP) as NCOP = COP/CCOP. The Carnot coefficient of performance is the ideal value with no losses. Hence, NCOP is a measure of how close to ideal has been achieved. We then wanted to know how the set of NCOP's should be combined. Fig. ! shows a schematic refrigerator with our definition of variables.

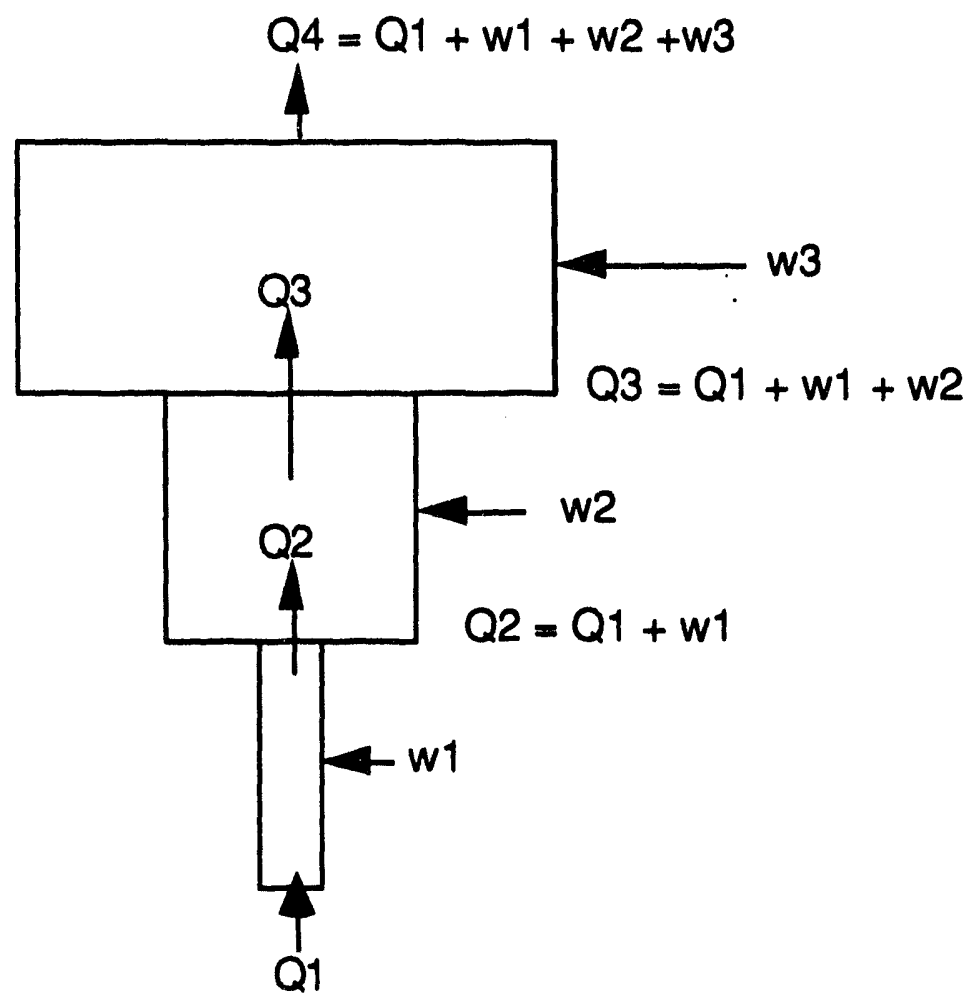

Figure (1) shows a sequence of individual sections, $\mathrm{i}=1,2,3$, connected in series and schematically varying in size as would be the case for a cryocooler. The purpose of the refrigerator is to remove a heat $Q_{1}$ and transfer it out of $Q_{3}$ by a sequence of processes each doing work of $w_{1}, w_{2}$, and $w_{3}$. The heat transferred stage to stage is augmented by the work performed in the previous stage. Thus the final stage must reject a total heat of $\mathrm{Q}_{4}=$ $\mathrm{Q}_{1}+\mathrm{w}_{1}+\mathrm{w}_{2}+\mathrm{w}_{3}$. The normalized COP is defined as $\eta_{\mathrm{i}}=\Gamma_{\mathrm{i}} / \Gamma_{\mathrm{c}}$ where $\Gamma_{\mathrm{i}}=\mathrm{Q}_{\mathrm{i}} / \mathrm{w}_{\mathrm{i}}$, the actual COP for each stage and $\Gamma_{C}$ is the Carnot COP. In CP I, $\eta_{j}$ is derived for $j$ stages of each of normalized COP, $\eta_{i}$ and where each stage has a constant temperature ratio of $T \mathrm{i} / \mathrm{T}(\mathrm{i}+1)=2$, so that $\Gamma_{\mathfrak{i}}=1$.

For the three such stages of Fig. 1, the NCOP becomes:

$$
\eta_{13}=7 / 3 \frac{\eta_{12} \eta_{3}}{\left(\eta_{12} / 3\right)+\eta_{3}+1}=\frac{7 \eta_{1} \eta_{2} \eta_{3}}{1+\eta_{1}+\eta_{2}+\eta_{3}+\eta_{1} \eta_{2}+\eta_{1} \eta_{3}+\eta_{2} \eta_{3}}
$$

with more complicated expressions for additional stages. We note that each combination of efficiencies is greater than the simple product, which is more favorable than one might have thought, and that if all $\eta_{i}=1$, then the combined efficiency is also equal to 1 as it should be. In addition this analysis gives assurance that one inefficient section does not disproportionately effect the over all efficiency. 
We now extend this concept of discreet stages to a continuous refrigerator of infinitesimal sections of temperature increment, $\delta \mathrm{T}$.

Let the heat transferred out at temperature $\mathrm{T}+\delta \mathrm{T}$ be $\mathrm{Q}_{\mathrm{T}+\delta \mathrm{T}}$. Then

$$
\mathrm{Q}_{\mathrm{T}+\delta \mathrm{T}}=\mathrm{Q}_{\mathrm{T}}+\mathrm{W}=\mathrm{Q}_{\mathrm{T}}\left(1+\frac{1}{\Gamma}\right)=\mathrm{Q}_{\mathrm{T}}\left(1+\frac{\delta \mathrm{T}}{\mathrm{T} \eta}\right)
$$

where $\eta$ is defined as above as the NCOP and $\Gamma$ is the CCOP. The entropy for the same infinitesimal change in temperature then becomes:

$$
S_{T+\delta T}=\frac{Q_{T}+\delta T}{T+\delta T}=Q_{T} \frac{\left(1+\frac{\delta T}{T \eta}\right)}{T+\delta T}=S_{T} \frac{\left(1+\frac{\delta T}{T}\right)}{\left(1+\frac{\delta T}{T}\right)}=S_{T}\left[1+\frac{\delta T}{T}\left(\frac{1}{\eta}-1\right)\right]
$$

or taking the limits:

$$
\frac{\mathrm{d} S}{\mathrm{dT}}=\frac{\mathrm{S}}{\mathrm{T}}\left(\frac{1}{\eta}-1\right) \text { and by integrating } \mathrm{S}=\mathrm{S}_{\mathrm{o}}\left(\frac{\mathrm{T}}{\mathrm{T}_{\mathrm{o}}}\right)^{\left(\frac{1}{\eta}-1\right)} \text {. }
$$

Thus for example, if $\eta=0.96$ and $T / T_{0}=75$, corresponding to a temperature ratio of $4 \mathrm{~K}$ to 300 $\mathrm{K}$, then $\mathrm{S} / \mathrm{S}_{\mathrm{o}}=1.2$; that is, the overall COP is only $20 \%$ greater than Carnot. This is a reasonable objective for the design of a regenerator, provided there is not some other larger penalty in efficiency required in order to remove this extra entropy.

The Entropy Source and Sinks in the Stirling Cycle

A Stirling cycle refrigerator removes heat or entropy from a cold load and deposits it in a hot reservoir as in Fig. 1. This processes of pumping entropy at the expense of work is described by the Sachur-Tetrode equation:

$$
S=N k\left\{\frac{3}{2}+\ln \left[\left(\frac{m}{h^{2} / 2 \pi}\right)^{3 / 2} \frac{(k T)^{5 / 2}}{P}\right]\right\} .
$$

Hence the energy removed from a cold terminal, $T \Delta S$, is just $N k T \ln \left(P_{\max } / P_{\min }\right)$, or NkT times the compression ratio, provided, of course that this compression (expansion) process occurs at constant T, i.e., isothermal. Since no entropy is removed except by expansion of compression, the regenerator, being constant volume, may modify the entropy within it, but not remove or add entropy from the outside. Therefore the extra entropy created in the regenerator must be removed at either end. The addition of entropy or removal of entropy from the system depends upon both the compression and expansion only. The Sachur-Tetrode equation above, applied to the Stirling engine, assumes an isothermal process, ( $T$ remains constant during a change in $P$.). It is thermal conduction that maintains the constant temperature that adds or removes entropy. During expansion at the cold end, heat must flow into the gas in order to maintain a constant temperature as the density is decreased, i.e., an increased entropy of the gas at the cold end because of the removal of entropy from a load. Conversely at the hot end, a compression would heat the gas, but conduction of heat into the reservoir reduces the entropy as the density is increased, thereby pumping entropy to the "universe". An ideal Stirling cycle compresses the gas only at the hot end and expands it only at the cold end. The transfer ideally occurs reversibly so that gas transferred from hot to cold and back again from cold to hot returns with the original entropy. The addition to this entropy by the transfer process alone within the regenerator is the subject of this paper. 
When compressed gas of entropy, $S_{1}$, enters the regenerator at the hot end, it exits the regenerator at the cold end at the ideal entropy of $S_{2}$, there has been added an entropy $\Delta S$ due to the imperfection of the regenerator. This extra entropy, $\Delta S_{+}=S-S_{0}$ of Eq. (4) must be removed from the gas by conduction to the cold reservoir. Only after this can expansion in the cold end remove entropy from the cold reservoir. Thus the total entropy that can be removed is reduced

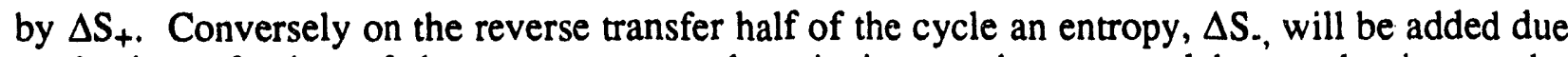
to the imperfection of the regenerator and again it must be removed by conduction to the reservoir before compression commences. In this later case, however, there is no penalty in PdV work required to remove the extra entropy at the hot end, provided the extra heat of the gas is removed before the start of compression. The change in temperature of the regenerator itself within a half cycle is second order in $\Delta S$, and so not considered. Instead the mean temperature of the regenerator will equilibrate such as to satisfy this condition for removing the extra entropy of the expanded state transfer to the hot end. This equilibrated state will add entropy to the subsequent compressed state transfer etc. Therefore both entropy gains will add to the inefficiency of the refrigerator, and so the total loss in COP will be assumed to be the sum of that occurring in both transfers.

We now wish to relate NCOP, $\eta$, to the extra entropy generated in the regenerator due to irreversible processes in the working fluid such as finite conduction and friction.

\section{Working Fluid Losses}

\section{Measure of Refrigeration}

The refrigeration work required to transfer an amount of heat $Q$ from $T$ to $T+d T$ is $\mathrm{Q}(\mathrm{dT} / \mathrm{T})$, proportional to $\mathrm{Q}$ and inversely proportional to the temperature past which it is transferred. Thus, a measure of refrigeration performance, or heat transfer difficulty is $\mathrm{Q} / \mathrm{T}$ or the entropy transferred per cycle or unit of time. The coefficient of performance is the ratio of heat transferred, $\mathrm{Q}$, to the PdV work required to transfer it. The Carnot COP is $\mathrm{T} / \mathrm{dT}$, while the actual COP is $Q /(P d V)$ so that the local efficiency is $Q d T /(T P d V)$. This is what we have defined as $\eta$. This efficiency is a measure of the extra PdV work required to transfer a given entropy $Q / T$ through dT. Losses (inefficiencies) are due to the production of entropy by friction and conduction. Our purpose is to minimize the entropy gain in each stage in the spirit of Minta and Smith (1984).

\section{Heat Exchange-Working Fluid to Wall}

The primary purpose of a regenerator is to exchange heat reversibly between a moving working fluid, i.e. gas for the Stirling cycle, and a stationary heat mass. The lack of perfect heat exchange leads to a mean temperature difference between the gas and the wall. The gradient of this temperature difference both drives the heat flow from the gas to the wall and also leads to an irreversible loss, that is, an entropy gain, or equivalently extra PdV work per cycle. This transverse heat flow takes place in the $y$ direction in a channel of width $W$ and in the axial direction due to a temperature gradient, $d T / d z=T_{z}{ }^{\prime}$ Here the characteristic length, $L=T / T_{z}$. The gas flows in the $z$ direction and the extent in the $x$ direction is the dimension, $B$. In addition there will an entropy gain due to thermal conduction along the length as well as friction with the walls, Fig. 2.

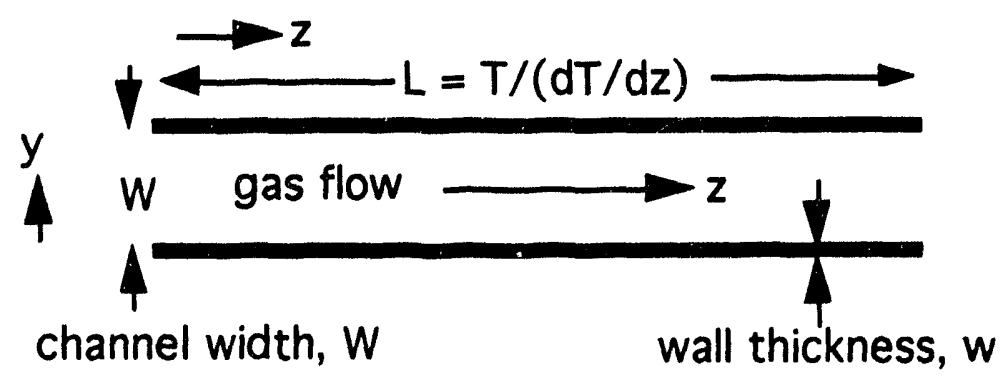


Figure (2) shows a idealized channel of width $W$ and temperature gradient, $T_{z}^{\prime}$. The gas flows in the $z$ direction, and the heat flows both transversely in the y direction to and from the walls as well as in the $z$ direction. The extent of the channel into the paper is $B$. The temperature is $T$ and the change in temperature is $\delta T$. The characteristic length $L=T / T_{z}^{\prime}$. The mean velocity, half cycle average is, $v$, the density $\rho$, and so the mass throughput is $B W v p=d M / d t$.

Heat flow generates entropy as analyzed by Kittel (1992). Here, instead of a heat flux that is harmonic in time we consider a steady, average value during each half of the cycle. For a temperature gradient, grad $(T)$, we have a heat flux ( $K \operatorname{grad}(T) A)$ through an area $A$ and over a distance $\Delta x$. (Here $\mathrm{x}$ is a generalized coordinate, not the direction $\mathrm{B}$.) The temperature goes from $\mathrm{T}$ to $\mathrm{T}-\mathrm{grad}(\mathrm{T}) \Delta \mathrm{x}$ so that the entropy change rate in a volume $\Delta \mathrm{V}=\mathrm{A} \Delta \mathrm{x}$ is:

$$
\Delta \dot{S}=\kappa(\nabla T) A\left(\frac{1}{T-\nabla T \Delta x}-\frac{1}{T}\right)=\kappa\left(\frac{\nabla T}{T}\right)^{2} \Delta V
$$

Assuming grad $(\mathrm{T})$ is independent of the coordinates in the $\mathrm{B}$ and $\mathrm{L}$ directions, we have

$$
\frac{\dot{S}}{\dot{M}}=\frac{B L}{B W v \rho} \int_{-W / 2}^{W / 2} k\left(\frac{\nabla T}{T}\right)^{2} d y
$$

We now must substitute for the two temperature gradients where the longitudinal one is $\Delta \mathrm{T} / \mathrm{L}$. Transversely, $\mathrm{T}$ is nearly parabolic, the deviation going from $\delta \mathrm{T}$ at the center to 0 at the wall, i.e. $\mathrm{T}(\mathrm{y})=\mathrm{T}+\delta \mathrm{T}\left(1-4 \mathrm{y}^{2} / \mathrm{w}^{2}\right)$ and so $\operatorname{grad} \mathrm{T}=-\delta \mathrm{T}\left(8 \mathrm{y} / \mathrm{w}^{2}\right)$. The net heat advection must equal the heat flow to the walls, so that

$$
\delta T=(1 / 2) \Delta T \frac{\rho v c_{p} W^{2}}{8 K L(W / 2)}
$$

and

$$
\left|\nabla T_{\perp}\right|=\Delta T \frac{\rho v c_{p} y}{\kappa L} .
$$

If we use the longitudinal and this transverse gradient in the integral of $\mathrm{Eq}(7)$, then the entropy generation becomes:

$$
\frac{\dot{S}}{\dot{M}}=\frac{\kappa}{v p L}\left(\frac{\Delta T}{T}\right)^{2}\left[1+\frac{1}{12}\left(\frac{\rho v c_{p}}{\kappa} W\right)^{2}\right]
$$

\section{Wall Friction}

We must now must add the entropy generated by the frictional work of the gas with the walls. The work is $\triangle P W \vee B$, generating an entropy $(\triangle P W \vee B) / T$ so that

$$
\frac{\dot{S}}{\dot{M}}=\frac{\Delta P}{\rho T}
$$


Poiseuille flow in a channel gives $\nabla P=\frac{\Delta P}{L}=\mu \frac{d^{2} v}{d y^{2}}$, which gives $v(y)=v_{\max }\left(1-4 y^{2} / w^{2}\right)$ and so $v_{\max }=\left(\mathrm{W}^{2} / 8\right)(\Delta \mathrm{P} / \mu \mathrm{L})$. If we average $\mathrm{v}(\mathrm{y})$ from 0 to $\mathrm{W} / 2$, then $\mathrm{v}=(2 / 3) \mathrm{v}_{\max }=(1 / 12) \mathrm{W}^{2}$ $(\Delta \mathrm{P} / \mu \mathrm{L})$. Then for the friction loss we have

$$
\frac{\dot{S}}{\dot{M}}=\frac{12 \mu \mathrm{Lv}}{\rho T W^{2}}
$$

\section{Combined Losses}

We must add this entropy generation to that associated with the heat flow of Eq. (10). In terms of these variables adding the three losses gives

$$
\sigma=\frac{\dot{S}}{\dot{M} c_{p}(\Delta T / T)}=\left[\frac{D}{L v}+\frac{v W^{2}}{12 L D}+\frac{12 L D v}{W^{2} c_{s}^{2}}\right]
$$

or ; fractional loss = parallel conduction + perpendicular conduction + friction

The left hand side of Eq. (13) is just the ratio of the entropy generated in losses to the useful entropy transferred through the regenerator. It is therefore just the fractional loss, or $\sigma$, for a given $\Delta T / T$ or $(1-N C O P)=1-\eta$. For small $\sigma$ this is $\sim(1 / \eta-1)$ of Eq. (4).

If we assume that the working fluid is helium at or below atmospheric pressure and for temperatures above $4 \mathrm{~K}$, then the diffusion coefficient, $\mathrm{D}$, can be give as $\left(D_{0} / P\right) T^{3 / 2}$ and similarly $c_{s}^{2}=T c_{o}{ }^{2}$ where $T$ is in units of $T_{0}$, the high temperature, so that the loss fraction becomes:

$$
\sigma=\frac{\dot{S}}{\dot{M} c_{p}(\Delta T / T)}=\left[\frac{D_{0} T^{3 / 2}}{P L v}+\frac{v P W^{2} T^{-3 / 2}}{12 L D_{0}}+\frac{12 L v D_{0} T^{1 / 2}}{W^{2} c_{0}^{2} P}\right]
$$

\section{Minimization of Losses}

In CP I we showed that if $\sigma$ were expressed in terms of the dimensionless variables, Reynolds number, Prandtl number and Mach number, that we can make this fractional loss arbitrarily small. However, in order to minimize the dead volume of the regenerator, the velocity should be as high as possible so that at a given frequency, the maximum volume is transferred within a cycle. On the other hand the heat capacity of the solid wall materials becomes so small at low temperature, $\propto \mathrm{T}^{2}$, that there is a strong motivation to increase the surface area at low temperature. An approximate minimum can be obtained by just setting all three losses equal. Then there is a particular scaling of $\mathrm{L} . \mathrm{W}$, and $\mathrm{v}$ that results in near constant loss fraction at lower temperatures, and increases the surface area at the expense of dead volume at low temperatures. The increased wall area is desirable in order to have enough accessable wall heat mass at low temperature. This particular scaling is:

$$
\mathrm{L}=\mathrm{L}_{\mathrm{O}} \mathrm{T}^{-1 / 2} ; \mathrm{W}^{2}=\mathrm{W}_{\mathrm{o}}^{2} \mathrm{~T}^{1 / 2} ; \mathrm{v}=\mathrm{v}_{\mathrm{o}} \mathrm{T}^{1 / 2} \text {. }
$$

When this scaling is substituted into Eq. (14), it results in the loss fraction :

$$
\sigma=\frac{\dot{S}}{\dot{M} c_{p}(\Delta T / T)}=\left[\frac{D_{0} T^{3 / 2}}{P_{L_{0}} v_{0}}+\frac{v_{0} P W_{0}^{2}}{12 L_{0} D_{0}}+\frac{12 L_{o} v_{0} D_{0}}{W_{o}^{2} c_{0}^{2} P}\right]
$$

or ; fractional loss $=$ parallel conduction + perpendicular conduction + friction 
The first term in the bracket, the loss due to longitudinal conduction in the gas, decreases as $\mathrm{T}^{3 / 2}$ and the remaining terms remain constant independent of temperature. One therefore neglects the first term, recognizing that the efficiency of the high temperature end will be reduced for the first factor of 2 change in temperature. (This can be compensated for by a small change in parameters for the first, highest temperature stage, without compromising the more demanding, low temperature design.) One then sets the last two terms equal for a minimum loss design. The result is;

$$
\frac{\mathrm{L}_{\mathrm{o}}}{\mathrm{W}_{\mathrm{o}}^{2}}=\frac{\mathrm{Pc}_{\mathrm{o}}}{12 \mathrm{D}_{\mathrm{o}}}=7.5 \times 10^{3} \mathrm{P} \quad \mathrm{cm}^{-1}
$$

where we have evaluated $c_{0}$ and $D_{0}$ at $300 \mathrm{~K}$ for helium. We note that this ratio is then independent of $\sigma$ and $v_{0}$, and demands a large aspect ratio for the channel dimensions. In addition using this result in Eq. (16) defines the velocity of the flow as:

$$
\mathrm{v}_{\mathrm{o}}=\sigma \mathrm{c}_{\mathrm{o}} / 2=4.5 \times 10^{4} \sigma \quad \mathrm{cm} \mathrm{s}^{-1} \text {. }
$$

for helium at 300 . In this case the Mach number is just half the fractional loss.

\section{Dead Volume}

The remaining constraint is the dead volume ratio, $\mathrm{R}$ Voldeacl. This ratio is a measure of the mass of the gas within the regenerator compared to the mass within the compression or expansion volumes at either end. It is therefore an integral property of the regenerator, but it affects the differential loss $\sigma$. For the scaling of Eq. (15), all the dead volume will be concentrated at the cold end (see Eq. (19) below). Then for the ideal refrigerator one would desire that the mass of gas transferred to the cold end expansion volume be larger than the gas remaining in the regenerator since the only useful cold mass that can remove entropy from a load or heat source is that within the cold expansion volume or equivalently that which is transferred from the regenerator. In addition the gas remaining in the regenerator adds to the entropy produced due to the heat transfer within the gas to and from the walls as from the second term in the brackets of Eqs. (13 or 16). There will also be a further entropy gain due to the finite heat mass of the walls and conduction within the wall material. Therefore we expect $\sigma$ to be effectively increased by roughly $\left(1+\mathrm{R}_{\text {Voldead }}\right)$. The dead volume ratio is

$$
R_{\text {voldead }}=\int_{0}^{z_{0}} \frac{d z}{v t}=\frac{(2 f) L_{0}}{\sigma c_{0}} \int_{T_{0}}^{T} T^{-2} d T=\frac{2 f L_{0}\left(T^{-1}-T_{0}^{-1}\right)}{\sigma c_{0}}=\frac{f W_{0}^{2} P\left(T^{-1}-T_{0}^{-1}\right)}{6 D_{0} \sigma}
$$

and so in order to maintain a small value of $\mathrm{R}$ Voldead at low temperature, we desire a low frequency, a small channel width, and modest loss fraction. These are all features that compete in the design of the regenerator.

\section{Wall Material Losses}

The wall acts as a heat source and sink by transferring a heat, $\Delta Q$ into and out of a uniform material of specific heat $c_{v w}$, and conductivity $\kappa_{w}$, within a half cycle time, $1 / 2 f$. The wall losses are made up of the time dependent heat flow in and out of, i.e., transverse to, the wall and the near steady state parallel conduction within the wall material. In addition if the heat mass of the wall either because of finite skin depth, $\lambda$, within the cycle time, or because the wall thickness, $w$, is in itself too small, then there will be an additional loss in the gas because the boundary temperature of the wall oscillates whereas a constant temperature boundary condition has been assumed. Treating the first two losses first, we note that they are entirely analogous to the entropy generated in the gas for nearly identical reasons. Similar to Eq. (7) the rate of entropy generation per unit of mass flow is calculated in a wall of width, w. Then for two walls the entropy production becomes: 


$$
\frac{\dot{S}}{\dot{M}}=\frac{2 B L}{B W v \rho} \int_{0}^{w} \kappa\left(\frac{\nabla T}{T}\right)^{2} d y
$$

As before we now must substitute for the two temperature gradients where the longitudinal one is $\mathrm{T} / \mathrm{L}$. Transversely, $\mathrm{T}$ is nearly gaussian which we approximate by parabolic, the deviation going from $\delta \mathrm{T}$ at the wall to nearly 0 at depths greater than $\lambda$, where $\lambda$, the thermal skin depth $=$ $\left[\left(k / c_{v w}\right)(1 / 2 f)\right]^{1 / 2}$, and so $\left.T(y)=T \pm \delta T_{w}\left(1-y^{2} / 2 \lambda^{2}\right)\right)$. Then $\operatorname{grad} T=-\delta T_{w}\left(y / \lambda^{2}\right)$. The net heat advection must equal the heat flow to the walls, where the heat advected is $\left(\rho v c_{p} W \Delta T\right)(1 / 2 f)$ and the heat flow within the walls in this time is $w_{L w} \delta T_{w}$.

so that

$$
\delta T_{w}=(1 / 2) \frac{\rho v c_{p} W \Delta T}{w L c_{v w}(2 f)}
$$

and

$$
\left|\nabla T_{\perp}\right|=\Delta T \frac{\rho v c_{p} W y}{2 w L K_{w}}
$$

where $c_{v w} \lambda^{2}(2 f)=\kappa_{w}$

If we use the longitudinal and this transverse gradient in the integral of $\mathrm{Eq}(20)$, then the entropy generation becomes:

$$
\begin{aligned}
& \frac{\dot{S}}{\dot{M} c_{p}(\Delta T / T)}=2\left(\frac{\Delta T}{T}\right)\left\{\frac{w K_{w}}{\dot{M} L}+\frac{\dot{M} w}{12 L K_{w}}\right] . \\
& \text { fractional loss = longitudinal conduction + transverse conduction }
\end{aligned}
$$

Longitudinal conduction loss takes place regardless of mass transfer, and so the fractional loss decreases with useful refrigeration and decreasing length. The transverse loss increases with mass transfer, but also decreases with length. Both losses are decreased by reducing the thickness or heat mass of the regenerator. However the limit of reduced heat mass is no regenerator at all.

\section{Finite Heat Mass}

To these losses we must consider the entropy generation in the gas due to the fluctuating wall boundary condition, $\pm \delta T_{w}$. The effect of this fluctuating boundary condition is not the obvious one of generating entropy within either the gas or wall material, because neither one experiences an increase in grad $T_{\perp}$. Instead, an increase in $\delta T_{w}$, which occurs due to insufficient heat mass of regenerator material, behaves in the limit as if there were negligible heat mass to the regenerator. The consequence is that the axial temperature gradient occurs alternately at one end and then the other as the heat is carried, without thermal lag to each isothermal compression and expansion volume. The entropy generated to first order will then correspond to that produced by a decrease in the effective value of $L$. This in turn affects the transverse heat conduction terms of boih Eq. (13 and 23), but without changing the friction term, proportional to $\mathrm{L}$ of the last term in Eq. (13). These effects are then an augmentation of losses already accounted for rather than a new and independent loss. On this basis, but without a detailed analysis, the finite heat mass condition becomes from Eq. (21): 


$$
\frac{\delta T_{w}}{\Delta T}=(1 / 2) \frac{\rho v c_{p} W}{w L c_{v w}(2 f)}=\frac{\dot{M} c_{p}}{\left(w L c_{v w}\right)(2 f)} \ll 1 .
$$

That is, the heat mass of the gas advected in a half cycle time must be small compared to the heat mass of the wall.

\section{Material Limits and Design Procedure}

The limiting useful thickness of the wall material is the thermal skin depth, $\lambda$, and so $w<$

$\lambda$. If $\lambda$ is substituted for $w$ in condition (24) then this determines the maximum heat mass of the wall and therefore determines whether a given wall material can satisfy the necessary boundary condition.

The design procedure therefore starts with the scaling conditions of Eq. (15) and the choice of a temperature ratio, presumably large like 75 in order to perform the cryogenic refrigerator function. Then a value of $\sigma$ must be chosen, which must be large, like 50 to 60 since the extra entropy is both generated and first transferred to the cold end and then transferred back to the hot end with further augmentation. . Also a pressure must be chosen, but in general since the objective is a temperature close to liquid helium temperature, the operating pressure can not be much greater than one atmosphere. One must then satisfy the dead volume relation of Eq. (19). In order for this ratio to be small enough, $<10 \%$ to $20 \%$ so that the other regenerator losses are not amplified, this sets a maximum frequency, $f$. With this value of frequency, the wall materials can be investigated. In general insulator materials like plastic or glass are satisfactory down to temperatures such as 10 to $20 \mathrm{~K}$, but below this temperature, higher conductivity and higher specific heat materials are desired. These conditions can be most readily satisfied by making the regenerator wall smooth but heterogeneous as for example of alternating conducting high heat capacity material and thermal insulator as in Colgate (1986). Such an analysis will be published in a subsequent paper, where one of us. SAC, plans an experimental test of the design

\section{Conclusion}

An analysis of the entropy generated by conduction and friction in a regenerator leads to a design that minimizes these losses as well as dead volume. This design maintains the Mach number of the flow constant, decreases the channel width as $T^{1 / 2}$ and increases the characteristic length, $\mathrm{L}=\mathrm{T}(\mathrm{dz} / \mathrm{dt})$ as $\mathrm{T}^{-1 / 2}$. Under these conditions the transverse conduction loss and friction loss remain constant and equal as a function of temperature and are therefore a relative minimum. The longitudinal loss decreases rapidly as $T^{3 / 2}$, and therefore can be neglected. These boundary values to the wall of a regenerator specify a range of wall material properties and frequency from which compromises must be chosen. This analysis also leads to a parallel channel design with small length and channel width.

\section{Acknowledgments}

Stirling A. Colgate is indebted to discussions with John Purcell and Ray Sarwinski of Advanced Cryomagnetics. Calculations by and discussions with Dodd Stacey of Creare, discussions with Ray Radebaugh and Jim Zimmerman of the USBS, Bascon Birmingham consultant., and Richard Colgate. This work has been supported by DOE, New Mexico Institute of Mining and Technology, and Colgate Thermodynamics.

\section{References}

1. Colgate, S.A., and Petschek, A.G., 1993, CEC,

1. Colgate, S.A., "Stirling Cycle Machine", 1986, US patent \# 4,619,112

2. Sullivan, D. B., Radebaugh, R., Daney, E. E., and Zimmerman, J. E., 1982, "An approach to optimization of low-power Stirling cryocoolers," Second Conference on Refrigeration for Cryogenic Sensors and Electrical Systems, NASA.

3. Walker, G., 1980, "Stirling Engineering," Oxford Univ. Press.

4. Uriel, D., Berchowitz, D.M., 1984, "Stirling Engine Analysis," Adam Hilger, Ltd., Bristol. 
5. Radebaugh, R. and Louie, B., 1984 "A simple first step to the optimization of regenerator geometry," Proc. of the Third Cryo-cooler Conference, NBS, CO, p. 177.

6. Coopage, J. E., and London, A. L., 1956, "Hleat transfer and flow friction characteristics of porous media," Chem. Eng. Prog. 52, p57-63.

7. Minta, M. and Smith, L. J., Jr., 1984, "An entropy flow optimization technique for helium liquification cycles," Advances in Cryoengineering 29, p469-487, R.W.

8. Stacy,D., McCormick, J.,and Wallis,P., 1992, "A Stirling Cryocooler for 4K Applications", 7th International Cryocooler Conferance, Santa Fe,NM , edited by Philips Laboratory, Kirtland AFB, NM 87117-5776, p. 444-459.

9. Kittel,P., "Enthalpy Flow Transition Losses in Regenerative Cryocoolers", 7th International Cryocooler Conferance, Santa Fe,NM , edited by Philips Laboratory, Kirtland AFB, NM 87117-5776, p. 1145.

10. Organ, Allan, J., "Thermodynamics and gas Dynamics of the Stirling Cycle Machine", Cambridge Univ. Press, 1992. 

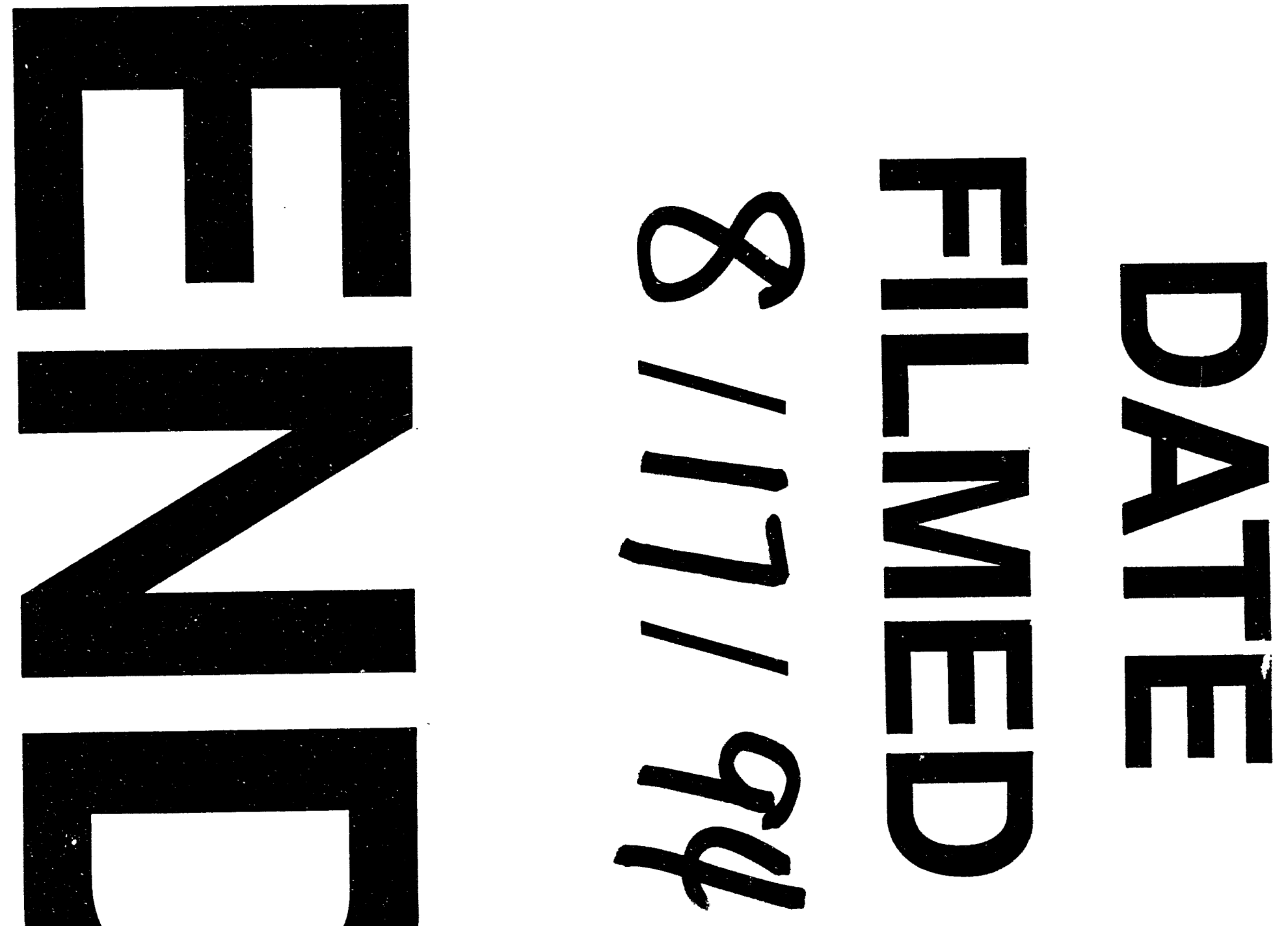
